\title{
User Resource Structure Design with Enhanced Diversity for OFDMA in Time-Varying Channels
}

\author{
Hlaing Minn \\ University of Texas at Dallas \\ Richardson, TX 75083 USA \\ Email: hlaing.minn@utdallas.edu
}

\author{
Chia-Chin Chong \\ DOCOMO Communications Laboratories USA, Inc. \\ 3240 Hillview Avenue, Palo Alto, CA 94304 USA \\ Email: cchong@docomolabs-usa.com
}

\begin{abstract}
It is a significant challenge to support high data rate services at high mobile speeds. In this paper, an efficient diversity-exploiting user resource structure, namely evolvingtype resource structure, for fast time-varying frequency-selective fading channel environments is designed. This newly proposed resource structure enjoys the advantages of both band-type and interleaved-type structures and avoids their disadvantages by exploiting both the multiuser diversity and the frequency diversity under fast time-varying channels. Simulation results corroborate the performance improvement of the new resource structure over the existing ones under various mobility scenarios.
\end{abstract}

Index Terms-Channelization, frequency diversity, multiuser diversity, OFDMA, resource structure, time-varying channel

\section{INTRODUCTION}

Multiuser and frequency diversities provide significant performance gains through proper link adaptation and resource allocation [1]-[6]. Essential to these performance gains is the knowledge of channel state information (CSI) for all users. Multiuser diversity implicitly requires time-varying channels, but in fast mobile channels, outdated CSI knowledge offsets the diversity gains [7], [8] resulting in a diversity-mobility tradeoff [9]. Diversity is crucial for counteracting channel fading, but an efficient diversity exploitation in fast timevarying channels is a challenge. This issue becomes more important as next generation wireless systems are aiming to support mobility up to $350 \mathrm{~km} / \mathrm{h} \mathrm{[10]}$.

Orthogonal frequency division multiple access (OFDMA) has been adopted in several wireless standards (e.g., 3GPP Long Term Evolution, 3GPP2 Ultra Mobile Broadband, and Mobile WiMAX), due to its low-complexity equalization and better resource resolution in the time-frequency grid. Resources can be allocated among users through a centralized or distributed approach. In general, better diversity gains may be realized in a centralized approach if more frequent CSIfeedback is provided. However, the overhead for acquiring channel knowledge and the related control/feedback signaling may not be affordable, especially for OFDMA in relatively fast time-varying channels. Several limited-feedback approaches have been recently proposed to address the overhead issue [11]-[14]. However, more frequent CSI updates imply a shorter frame length which could results in a significant increase in overhead ratio, and this overall overhead cost has not been taken into consideration in existing diversity-exploiting techniques. To alleviate this overhead issue, distributed access schemes have been recently proposed in [15]-[17] which can achieve multiuser diversity gains. However, these papers consider quasi-static channels only, and will be affected by time-varying channels.

Numerous resource allocation algorithms and user resource structures for OFDMA systems have been reported in the literature, each with different form of diversity exploitation. For instance, [18], [19] exploit multiuser diversity in their resource allocation or scheduling schemes through perfect or partial CSI. However, these works consider quasi-static or very slowly time-varying channels. The methods in [8], [18], [20] incorporate channel time-variations in their resource allocation assuming known Doppler spread, but they still suffer from performance degradation at high mobile speeds, and can also be affected by channel time-correlation model mismatches.

Despite several variations of the terminologies, the existing user resource structures can be classified into two main categories, namely, band-type and interleaved-type. The bandtype structure aims to exploit multiuser diversity in which the subcarriers of a user are adjacently located. However, in fast time-varying channels the channel allocated to a user may well be in deep fade at later part of the frame as illustrated in Fig. 1(a) causing a significant performance degradation. To alleviate this adverse deep fade, a shorter frame length and a more frequent CSI-feedback can be resorted, with the price of substantial throughput loss due to the associated large overhead. An alternative solution is to deploy the interleavedtype structure in which the subcarriers of a user are spread out across the whole band and interleaved with those of the other users. A user may have either all non-contiguous subcarriers or several non-adjacent groups of a few contiguous subcarriers which are spread out across the entire band. In a more general setup, the locations of the assigned subcarriers may also be shifted from symbol to symbol in a systematic way across all users. The interleaved-type tackles the deep fade problem through frequency diversity, but multiuser diversity gain is lost, as shown in Fig. 1(b).

This paper considers a different angle for diversity exploitation, namely user resource structure design (or user channelization). Existing resource allocation algorithms which exploit multiuser diversity can be applied over the proposed resource structures. We consider delay-constrained scenarios 


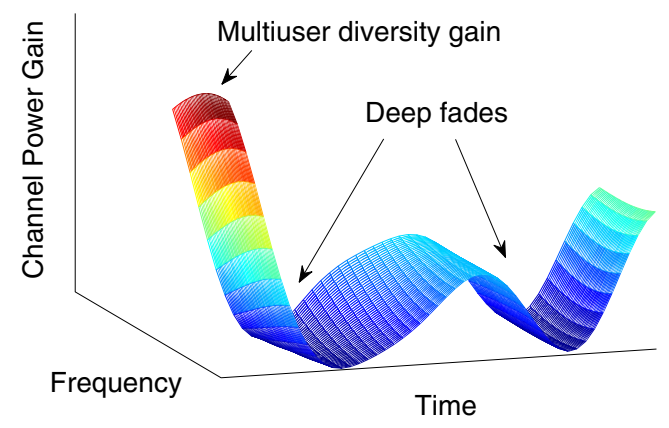

(a) Band-type resource structure.

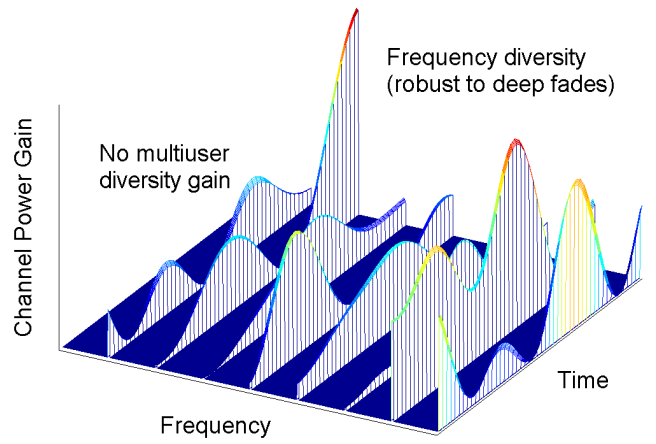

(b) Interleaved-type resource structure.

Fig. 1. An example of the channels experienced by (a) band-type, and (b) interleaved-type resource structures, respectively.

such that diversity exploitation across different frames are not allowed, while mobility can be as high as $350 \mathrm{~km} / \mathrm{h}$. In particular, an evolving-type user resource structure is proposed for multiuser systems in fast time-varying frequency-selective fading channels. The proposed resource structure exploits the multiuser diversity, frequency diversity and time diversity within the frame better than the existing resource structures.

The rest of the paper is organized as follows: Section II describes the proposed user resource structure design. Section III presents performance comparison of different resource structures under multipath fading channels with various mobile speeds. Finally, Section IV provides conclusions.

\section{Proposed User Resource Structure Design}

\section{A. Scheme Description}

The essence of the proposed approach is the introduction of a new degree of freedom in term of resource structuring in order to capture the best diversity gains in multiuser timevarying frequency-selective fading channels. When some CSI knowledge is available at the beginning of the transmission, significant multiuser diversity gain can be realized with proper resource allocation and structuring (e.g., band-type structure) for earlier part of the transmission frame. When the CSI

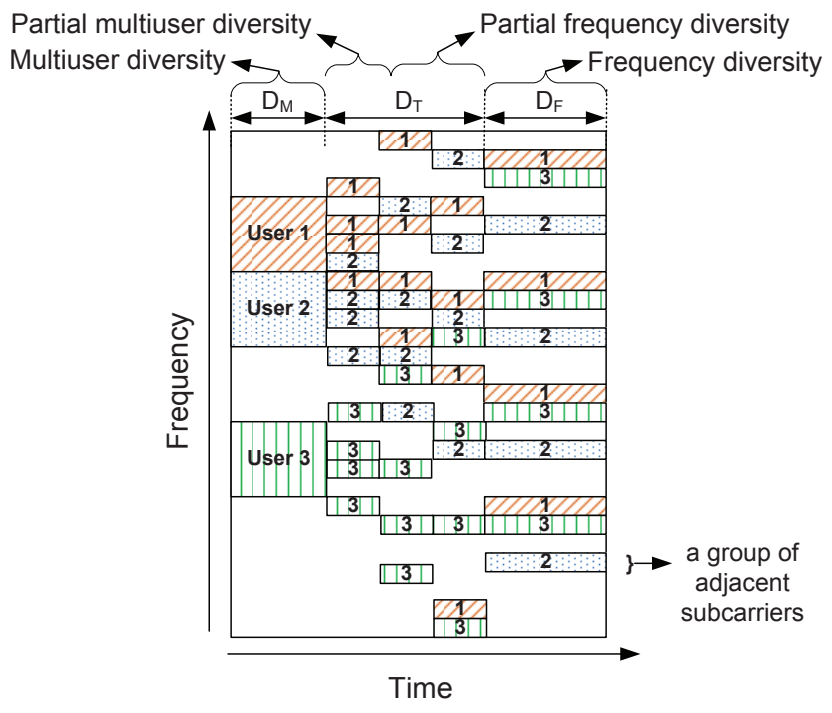

Fig. 2. An illustration of the proposed evolving resource structure.

at later part of the frame becomes less correlated with the a priori CSI knowledge at the beginning of the frame, using the same resource structure will not provide multiuser diversity gain and will be subject to deep channel fading. The interleaved-type resource structure possesses robustness against frequency-selective deep fading, however, it cannot exploit multiuser diversity. The proposed resource structure starts with the band-type at the beginning of the transmission frame (achieving multiuser diversity gain) and gradually evolve into the interleaved-type within the transmission frame (achieving frequency diversity or robustness against frequency-selective deep fading at later part of the frame). An example of the proposed evolving resource structure is illustrated in Fig. 2.

The proposed evolving resource structure can be divided into three phases: a) multiuser diversity exploitation phase (a duration of $D_{M}$ OFDM symbol periods); b) transition phase from multiuser diversity to frequency diversity (a duration of $D_{T}$ OFDM symbol periods); and c) frequency diversity exploitation phase (a duration of $D_{F}$ OFDM symbol periods). The early part of the transition phase enjoys partial multiuser diversity while its remaining part achieves partial frequency diversity. In general, the durations of these phases depend on the mobile speed, channel correlation characteristics, carrier frequency, symbol duration, and frame length.

\section{B. Design Criteria}

Our design approach and criteria are described below:

- The available $N$ subcarriers within one OFDM symbol are first divided into $K$ basic blocks of $N / K$ consecutive subcarriers each. Note that $N / K$ should be much smaller than the number of subcarriers within the channel coherence bandwidth, but a very small value of $N / K$ (say 1 or 2) is undesirable since it will be more susceptable to multiuser interferences caused by frequency offsets. The $K M$ basic resource blocks within a transmission frame of $M$ OFDM symbols will be channelized into 
$U$ subchannels of the same resource amount. ${ }^{1}$

- Each subchannel starts from a band-type structure defined by the consecutive $K / U$ basic blocks in each symbol and gradually evolves into an interleaved type where the $K / U$ blocks in each symbol of every subchannel are spread out across the entire band with approximately equal spacing (see Fig. 2). This evolving structure for the $u$ th subchannel can be defined by the basic block index set across time which is represented by a $(K / U \times M)$ matrix $\boldsymbol{\Lambda}_{u}$ in which the $m$ th column denoted by $\Lambda_{u, m}$ corresponds to the basic block index set for the $m$ th OFDM symbol. Prior to the design of $\left\{\boldsymbol{\Lambda}_{u}\right\}$, we first obtain a template of evolving block index sets $\left\{\boldsymbol{J}_{u, d}\right.$ : $u=1,2, \ldots, U ; d=1,2, \ldots, D\}$ such that:

1) $\boldsymbol{J}_{u_{1}, d}$ and $\boldsymbol{J}_{u_{2}, d}$ are disjoint for all $u_{1} \neq u_{2}$.

2) $\left\{\boldsymbol{J}_{u, 1}\right\}$ are of band-type i.e., each of them consists of consecutive basic block indexes.

3) $\left\{\boldsymbol{J}_{u, d+1}\right\}$ is a spreadout version of $\left\{\boldsymbol{J}_{u, d}\right\}$ to both sides in a circular manner. Neither too slow nor too fast expansion are desirable. The rule of thumb is that the span of $\left\{\boldsymbol{J}_{u, d+1}\right\}$ is approximately constrained to be within $1.3-1.5$ times the span of $\left\{\boldsymbol{J}_{u, d}\right\}$. The expansion ratio need not to be constant, and currently it is determined heuristically.

4) This spreading-out procedure continues until the basic block indexes of each subchannel become approximately (cyclically) equally spaced across the entire band. This will also determine the value of $D$.

In other words, there are $D$ distinct stages (i.e., different sets of block indexes) in the evolution from the band-type to the interleaved-type. The first stage is the band type itself, and the $D$ th stage is the interleaved type. Note that, the number of repeated OFDM symbols in each distinct evolving stage may not be the same.

- Let us denotes the number of OFDM symbols in the first stage as $\alpha_{1}$, while the number of OFDM symbols in each stage from the 2 nd to $(D-1)$ th stage is kept the same and denoted by $\alpha_{2}$. If $M>\alpha_{1}+(D-2) \alpha_{2}$, where $M$ is the total number of OFDM symbols per packet (or frame), there will be $M-\alpha_{1}+(D-2) \alpha_{2}$ symbols at the $D$ th stage, and the resource structure has fully evolved. If $M<\alpha_{1}+(D-2) \alpha_{2}$, the evolving structure will not reach to the (complete) interleaved-type, and the resource structure has just partially evolved.

- The designs of $\alpha_{1}$ and $\alpha_{2}$ can be based on the time intervals for which the correlation coefficient $\rho$ of the channel response reaches to some values $\rho_{1}$ and $\rho_{2}$, respectively. For instance, if $\rho_{1}=0.9$ and $\rho_{2}=0.5$ are used, $\alpha_{1}$ can be designed as:

$$
\begin{aligned}
& \alpha_{1}=N_{1}-1, \\
& \text { with } \quad N_{1} \triangleq \operatorname{round}\left(T_{90} / T_{\text {sym }}\right),
\end{aligned}
$$

\footnotetext{
${ }^{1}$ Each user may be assigned to one or more subchannels according to its data rate requirement and its CSI, but this resource allocation is out of the scope of this paper.
}

where round $(\cdot)$ denotes the rounding up, down, or to the nearest integer (up to the designer's choice), $T_{X}$ is the time interval between $\rho=1$ and $\rho=X / 100$, and $T_{\text {sym }}$ is the OFDM symbol duration including cyclic prefix (CP) or zero-padded guard interval. Note that the CSI delay of one OFDM symbol is incorporated in (1) by -1 . Similarly, $\alpha_{2}$ can be designed as:

$$
\begin{array}{ll} 
& \alpha_{2}=\left(N_{2}-N_{1}\right) /(D-2), \\
\text { with } & N_{2} \triangleq \operatorname{round}\left(T_{50} / T_{\text {sym }}\right) .
\end{array}
$$

The rationale of this design can be justified as follows. When $\rho \geq \rho_{1}=0.9$, the channel gains are highly correlated and hence the proposed structure maintains the same band-type within $\alpha_{1}$ symbols in order to exploit the multiuser diversity. When $\rho$ drops to $\rho_{2}=0.5$, the CSI knowledge at the beginning of the packet becomes outdated. At that time interval, the resource structure should exploit frequency diversity (as exploiting multiuser diversity is infeasible) and hence it should reach to an interleaved-type structure. Correspondingly, there are $\left(N_{2}-N_{1}\right)$ symbols for the middle $(D-2)$ stages which give $\alpha_{2}$ as shown in (3). While $\rho_{1}=0.9$ and $\rho_{2}=0.5$ are recommended, their settings are up to the designer.

\section{Case Study}

As a case study, let us consider a $N=256$ subcarriers OFDM system with $K=32$ basic blocks per symbol where each basic block consists of $N / K=8$ consecutive subcarriers. Suppose there are $U=4$ subchannels, then each subchannel has $K / U=8$ basic blocks per symbol. We can then heuristically construct several variations of the evolving block index matrices $\left\{\boldsymbol{J}_{\boldsymbol{u}}\right\}$. An example of $\left\{\boldsymbol{J}_{u}\right\}$ with $D=5$ distinct stages is given below:

$$
\boldsymbol{J}_{1}^{T}=\left[\begin{array}{rrrrrrrr}
1 & 2 & 3 & 4 & 5 & 6 & 7 & 8 \\
31 & 1 & 3 & 4 & 5 & 6 & 8 & 10 \\
29 & 31 & 1 & 3 & 6 & 8 & 10 & 12 \\
26 & 29 & 32 & 3 & 6 & 9 & 12 & 15 \\
22 & 26 & 30 & 2 & 6 & 10 & 14 & 18
\end{array}\right],
$$

$$
\boldsymbol{J}_{2}^{T}=\left[\begin{array}{rrrrrrrr}
9 & 10 & 11 & 12 & 13 & 14 & 15 & 16 \\
7 & 9 & 11 & 12 & 13 & 14 & 16 & 18 \\
5 & 7 & 9 & 11 & 14 & 16 & 18 & 20 \\
2 & 5 & 8 & 11 & 14 & 17 & 20 & 23 \\
3 & 7 & 11 & 15 & 19 & 23 & 27 & 31
\end{array}\right]
$$

$$
\begin{aligned}
\boldsymbol{J}_{3}^{T}= & {\left[\begin{array}{rrrrrrrr}
17 & 18 & 19 & 20 & 21 & 22 & 23 & 24 \\
15 & 17 & 19 & 20 & 21 & 22 & 24 & 26 \\
13 & 15 & 17 & 19 & 22 & 24 & 26 & 28 \\
10 & 13 & 16 & 19 & 22 & 25 & 28 & 31 \\
8 & 12 & 16 & 20 & 24 & 28 & 32 & 4
\end{array}\right], } \\
\boldsymbol{J}_{4}^{T}= & {\left[\begin{array}{rrrrrrrr}
25 & 26 & 27 & 28 & 29 & 30 & 31 & 32 \\
23 & 25 & 27 & 28 & 29 & 30 & 32 & 2 \\
21 & 23 & 25 & 27 & 30 & 33 & 2 & 4 \\
18 & 21 & 24 & 27 & 30 & 1 & 4 & 7 \\
13 & 17 & 21 & 25 & 29 & 1 & 5 & 9
\end{array}\right], }
\end{aligned}
$$


TABLE I

AN EXAMPLE DESIGN OF $\alpha_{1}$ AND $\alpha_{2}$ BASED ON $\rho_{1}=0.9$ AND $\rho_{2}=0.5$ IN A CHANNEL WITH THE CLARK-JAKES' DOPPLER SPECTRUM

\begin{tabular}{||c|c|c||}
\hline Mobile speed $v(\mathrm{~km} / \mathrm{h})$ & $\alpha_{1}$ & $\alpha_{2}$ \\
\hline 3 & 141 & 65 \\
\hline 20 & 20 & 9 \\
\hline 50 & 7 & 4 \\
\hline 80 & 4 & 2 \\
\hline 120 & 2 & 2 \\
\hline 200 & 2 & 1 \\
\hline 350 & 1 & 1 \\
\hline
\end{tabular}

where $(\cdot)^{T}$ denotes the transpose operator.

Table I presents values of $\alpha_{1}$ and $\alpha_{2}$ based on $\rho_{1}=0.9$ and $\rho_{2}=0.5$ (or equivalently $T_{90}$ and $T_{50}$ ) in a channel having the Clark-Jakes' Doppler spectrum with a maximum Doppler frequency determined by the mobile speed. The system parameters are the same as those defined in Section III-A.

Given $M,\left\{\boldsymbol{J}_{u}\right\}, \alpha_{1}$ and $\alpha_{2}$, the block index sets $\Lambda_{u, m}$ can be easily obtained for different values of the mobile speed $v$. For example, using $M=32$, and $\left\{\boldsymbol{J}_{u}\right\}, \alpha_{1}$ and $\alpha_{2}$ given above, we have:

$$
\begin{aligned}
3 \mathrm{~km} / \mathrm{h} \Rightarrow \Lambda_{u, m}=\boldsymbol{J}_{u, 1}, \forall m \in\{1, \ldots, 32\} \\
20 \mathrm{~km} / \mathrm{h} \Rightarrow \Lambda_{u, m}= \begin{cases}\boldsymbol{J}_{u, 1}, & m=1, \ldots, 20 \\
\boldsymbol{J}_{u, 2}, & m=21, \ldots, 29 \\
\boldsymbol{J}_{u, 3}, & m=30, \ldots, 32\end{cases} \\
50 \mathrm{~km} / \mathrm{h} \Rightarrow \Lambda_{u, m}= \begin{cases}\boldsymbol{J}_{u, 1}, & m=1, \ldots, 7 \\
\boldsymbol{J}_{u, 2}, & m=8, \ldots, 11 \\
\boldsymbol{J}_{u, 3}, & m=12, \ldots, 15 \\
\boldsymbol{J}_{u, 4}, & m=16, \ldots, 19 \\
\boldsymbol{J}_{u, 5}, & m=20, \ldots, 32\end{cases} \\
120 \mathrm{~km} / \mathrm{h} \Rightarrow \Lambda_{u, m}= \begin{cases}\boldsymbol{J}_{u, 1}, & m=1,2 \\
\boldsymbol{J}_{u, 2}, & m=3,4 \\
\boldsymbol{J}_{u, 3}, & m=5,6 \\
\boldsymbol{J}_{u, 4}, & m=7,8 \\
\boldsymbol{J}_{u, 5}, & m=9, \ldots, 32 .\end{cases}
\end{aligned}
$$

Those for other mobile speeds and frame lengths can easily be obtained similarly. The proposed evolving resource structure for the speed of $50 \mathrm{~km} / \mathrm{h}$ mentioned above has a multiuser diversity exploitation phase of $D_{M}=7$ symbols, a $D_{T}=12$ symbols transition phase from multiuser diversity to frequency diversity exploitation, and a frequency diversity exploitation phase of $D_{F}=13$ symbols (c.f. Fig. 2).

\section{iII. Performance Evaluation}

\section{A. Simulation Parameters}

Here we consider an OFDMA system with $N=256$ subcarriers and BPSK modulation. The carrier frequency is $2.5 \mathrm{GHz}$, the subcarrier spacing is $10.9375 \mathrm{kHz}$, and the $\mathrm{CP}$ length is $1 / 8$ of the useful symbol duration. Frame lengths of $M=16$ and 32 symbols are considered. The multipath channel has 16 uncorrelated Rayleigh fading taps having an exponential power delay profile with a $3 \mathrm{~dB}$ per tap decay factor. The channel gains are assumed to be constant ${ }^{2}$ within one symbol duration but vary from symbol to symbol according to the Clark-Jakes' Doppler spectrum. The mobile speeds as given in Table I are evaluated which are similar to the speeds targeted in the IMT-Advanced system specifications [10]. A block interleaver is applied and the interleaving is within a frame rather than an OFDM symbol. The industrial standard convolutional code with rate $1 / 2$, constraint length 7 , and generator matrix $[133,171]$ is applied across subcarriers. We assume perfect synchronization and channel estimation, and use the Viterbi decoding.

For the proposed evolving resource structure, the same setting as described in Section II-C will be used. For the band-type structure, each subchannel consists of 8 consecutive basic blocks, while for the interleaved-type structure, there are 8 equally spaced basic blocks across the entire band, and these block locations remain unchanged within the frame. We assume that channel knowledge at the beginning of the frame is available for the resource allocation. For the proposed structure or the band-type structure, the allocated resource in the first symbol has the best channel gains for the user. For the interleaved-type structure, resource allocation is performed randomly as there is no difference.

\section{B. Simulation Results and Discussion}

The plots of BER versus mobile speed at signal-to-noise ratio per bit $E_{b} / N_{0}$ of $0 \mathrm{~dB}$ and $5 \mathrm{~dB}$ are presented in Figs. 3(a) and 3(b) for a frame length of 16 symbols, and in Figs. 4(a) and 4(b) for a frame length of 32 symbols, respectively. From these figures, we can see that at a very low mobile speed, the proposed structure has the same performance as the band-type structure while the interleaved-type structure experiences a performance loss. When the mobile speed becomes very high, the performance of the band-type structure is significantly affected, while the interleaved-type structure enjoys a good performance. Notably, the proposed structure even outperforms the interleaved-type in the high mobile speed scenario due to the multiuser diversity gain at the beginning of the frame. At medium mobile speeds, the proposed structure enjoys performance gains over both existing resource structures due to its better diversity exploitability.

The BER versus $E_{b} / N_{0}$ plots for a frame length of 16 symbols are shown in Figs. 5, 6, and 7 for mobile speeds of $3 \mathrm{~km} / \mathrm{h}, 80 \mathrm{~km} / \mathrm{h}$, and $350 \mathrm{~km} / \mathrm{h}$, respectively. The advantage of the proposed structure over the interleaved-type structure is more significant at a slow mobile speed, while that over the band-type structure is more pronounced with high mobility.

Note that interleaving within the frame rather than within one OFDM symbol provides a better time diversity. Due to the limited space and being a well-known fact, the performance comparison in this aspect is omitted. In fact, all three resource structures exploit this time diversity. Due to the multiuser diversity, the band-type structure yields a better performance

\footnotetext{
${ }^{2}$ The channel variations within an OFDM symbol can be handled by means of channel estimate interpolation and intercarrier interference cancelation.
} 


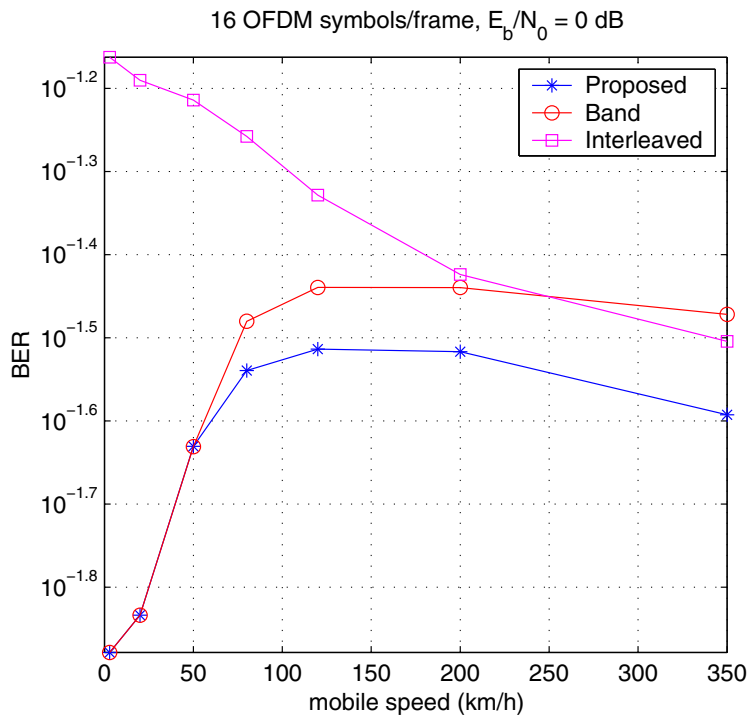

(a) $E_{b} / N_{0}=0 \mathrm{~dB}$.

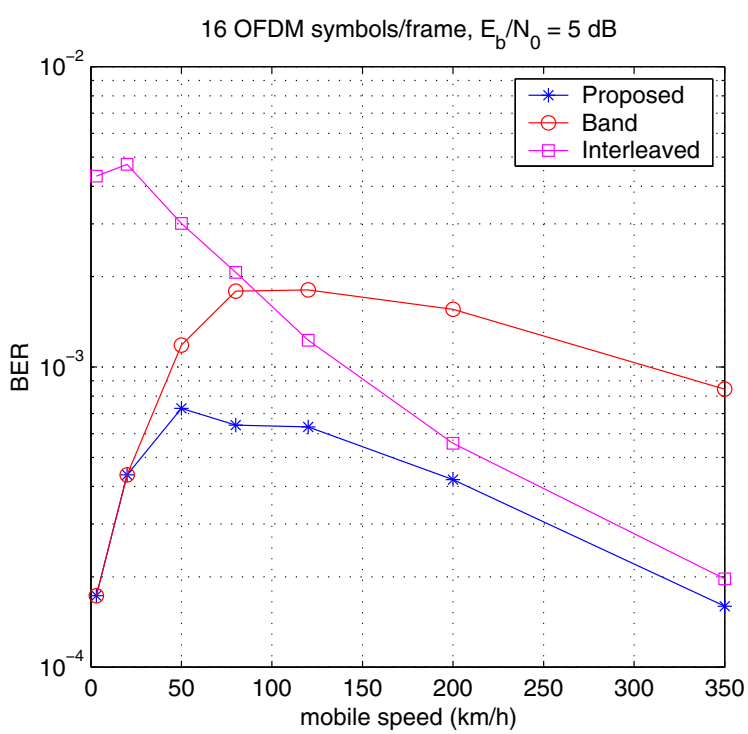

(b) $E_{b} / N_{0}=5 \mathrm{~dB}$

Fig. 3. BER versus mobile speed performance comparison of different resource structures with a frame length of 16 symbols.

than the interleaved-type structure in slow-varying channels. But in fast time-varying channels, the frequency diversity of the interleaved-type structure gives a better result than the band-type structure. By exploiting both multiuser diversity and frequency diversity, the proposed evolving resource structure outperforms both existing resource structures.

\section{Conclusions}

Efficient diversity exploitation in fast time-varying frequency-selective fading channels is a challenging task due to the channel knowledge requirement for the link adaptation and the outdated channel knowledge caused by the fast channel time-variations. We introduce a new angle for a

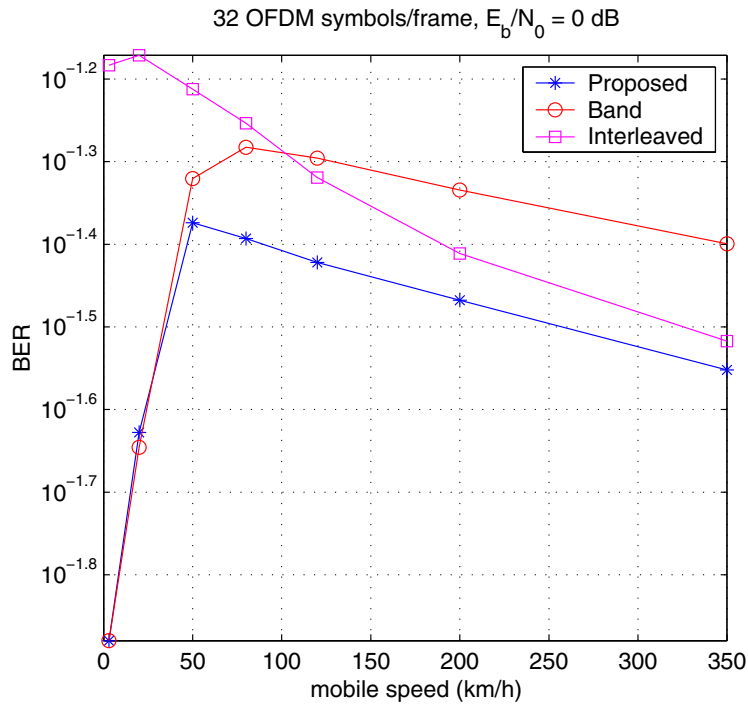

(a) $E_{b} / N_{0}=0 \mathrm{~dB}$.

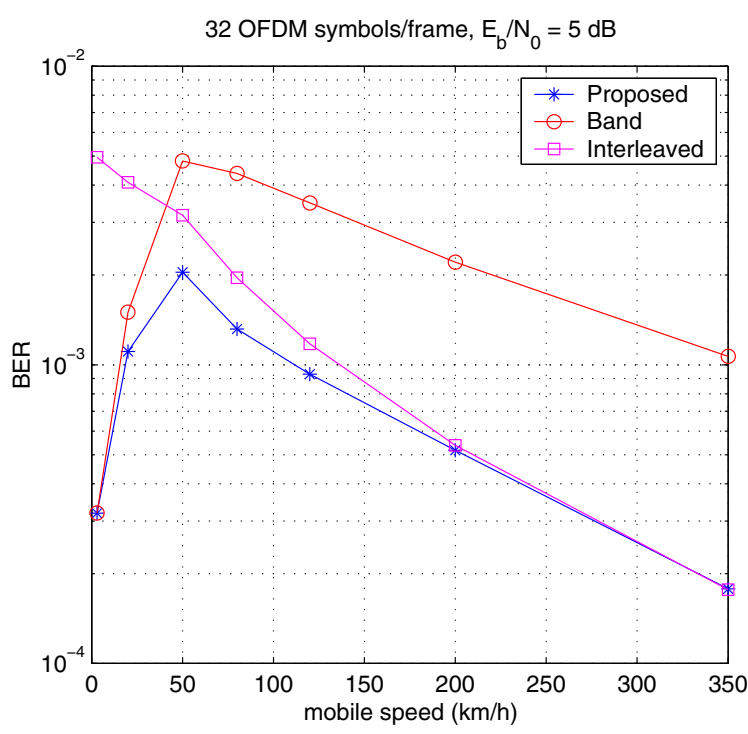

(b) $E_{b} / N_{0}=5 \mathrm{~dB}$.

Fig. 4. BER versus mobile speed performance comparison of different resource structures with a frame length of 32 symbols.

better diversity exploitation in such environments by means of the user resource structure design. We have presented a novel user resource structure design for OFDMA systems in mobile broadband wireless channels. Due to its better exploitability of multiuser diversity and frequency diversity, the proposed evolving resource structure outperforms the existing band-type and interleaved-type resource structures across the mobile speeds of interest. The proposed structure can be applied in both uplink and downlink with centralized as well as distributed access schemes.

\section{REFERENCES}

[1] R. Knopp and P. Humblet, "Information capacity and power control in single-cell multiuser communications," in Proc. IEEE Int. Conf. on 


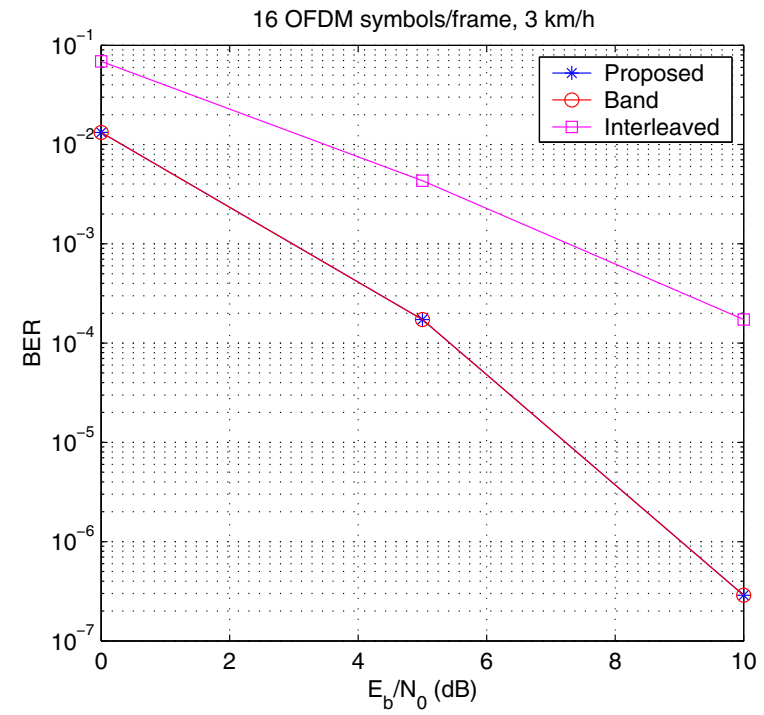

Fig. 5. BER versus $E_{b} / N_{0}$ performance comparison of different resource structures with a frame length of 16 symbols at a mobile speed of $3 \mathrm{~km} / \mathrm{h}$.

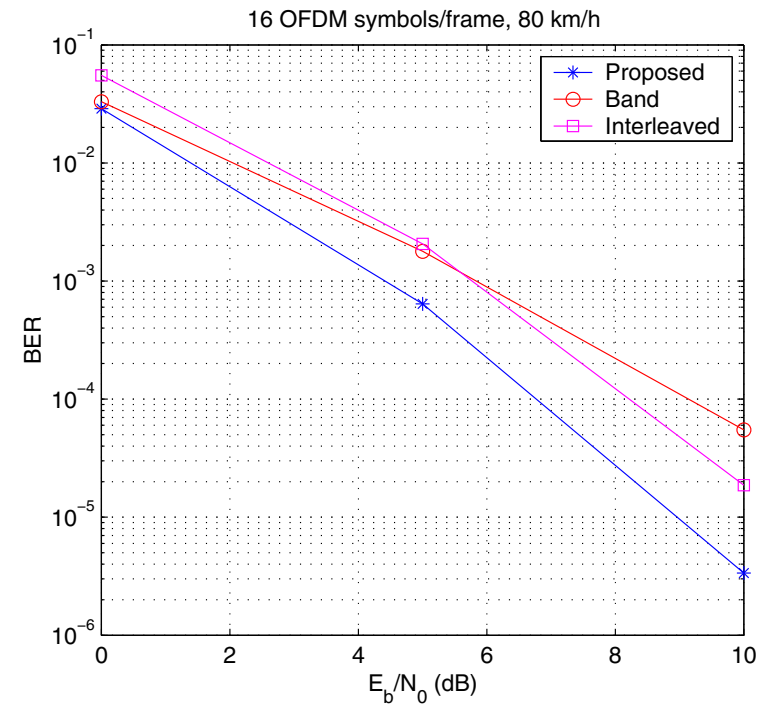

Fig. 6. BER versus $E_{b} / N_{0}$ performance comparison of different resource structures with a frame length of 16 symbols at a mobile speed of $80 \mathrm{~km} / \mathrm{h}$.

Commun., vol. 1, Ulm, Germany, Jun. 1995, pp. 331-335.

[2] D. N. C. Tse, "Optimal power allocation over parallel gaussian broadcast channels," in Proc. IEEE Int. Symp. on Inform. Theory, Ulm, Germany, Jun. 1997, p. 27.

[3] K.-D. Lee and V. Leung, "Fair allocation of subcarrier and power in an OFDMA wireless mesh network," IEEE J. Sel. Areas Commun., vol. 24, no. 11, pp. 2051-2060, Nov 2006.

[4] G. Li and H. Liu, "Downlink radio resource allocation for multi-cell OFDMA system," IEEE Trans. Wireless Commun., vol. 5, no. 12, pp. 3451-3459, Dec 2006.

[5] N. Ermolova and B. Makarevitch, "Low complexity adaptive power and subcarrier allocation for OFDMA," IEEE Trans. Wireless Commun., vol. 6, no. 2, pp. 433-437, Feb 2007.

[6] S. J. Lee, "Trade-off between frequency diversity gain and frequency selective scheduling gain in OFDMA systems with spatial diversity," IEEE Commun. Lett., vol. 11, no. 6, pp. 507-509, Jun 2007.

[7] D. Goeckel, "Adaptive coding for time-varying channels using outdated fading estimates," IEEE Trans. Commun., vol. 47, no. 6, pp. 844-855,

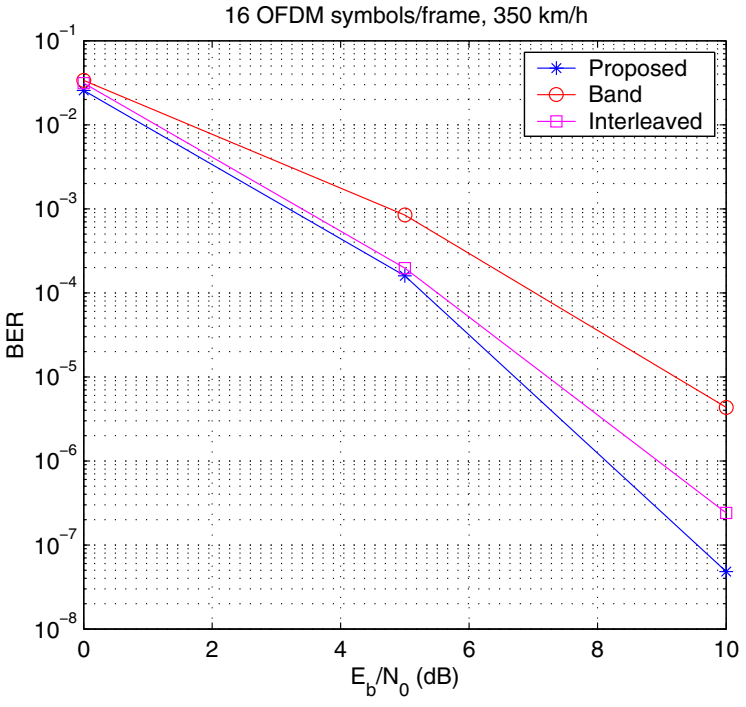

Fig. 7. BER versus $E_{b} / N_{0}$ performance comparison of different resource structures with a frame length of 16 symbols at a mobile speed of $350 \mathrm{~km} / \mathrm{h}$.

Jun 1999.

[8] F.-S. Chu and K.-C. Chen, "Fair adaptive radio resource allocation of mobile OFDMA," in Proc. IEEE Int. Symp. on Personal, Indoor and Mobile Radio Commun., Helskini, Finland, Sep. 2006, pp. 1-5.

[9] D. Piazza and L. B. Milstein, "Analysis of multiuser diversity in timevarying channels," IEEE Trans. Wireless Commun., vol. 6, no. 12, pp. 4412-4419, Dec 2007.

[10] Draft Report on Requirement Related to Technical Performance for IMT-Advanced 'Radio Interface(s) [IMT.TECH], ITU Std. Document 5D/TEMP/89R1-E, Jun 2008.

[11] G. Jongren and M. Skoglund, "Quantized feedback information in orthogonal space-time block coding," IEEE Trans. Inf. Theory, vol. 50, no. 10 , pp. $2473-2486$.

[12] V. Lau, Y. Liu, and T.-A. Chen, "On the design of MIMO blockfading channels with feedback-link capacity constraint," IEEE Trans. Commun., vol. 52 , no. 1 , pp. 62-70, Jan 2004.

[13] J. Choi and R. W. Heath, "Interpolation based transmit beamforming for MIMO-OFDM with limited feedback," IEEE Trans. Signal Process., vol. 53, no. 11, pp. 4125-4135, Nov 2005.

[14] T. Pande, D. J. Love, and J. V. Krogmeier, "Reduced feedback MIMOOFDM precoding and antenna selection," IEEE Trans. Signal Process., vol. 55, no. 5, pp. 2284-2293, May 2007.

[15] X. Qin and R. Berry, "Distributed approaches for exploiting multiuser diversity in wireless networks," IEEE Trans. Inf. Theory, vol. 52, no. 2, pp. 392-413, Feb 2006.

[16] Y. Xue, T. Kaiser, and A. B. Gershman, "Channel-aware ALOHA-based OFDM subcarrier assignment in single-cell wireless communications," IEEE Trans. Commun., vol. 55, no. 5, pp. 953-962, May 2007.

[17] D. Wang, H. Minn, and N. Al-Dhahir, "A distributed opportunistic access scheme and its application to OFDMA systems," IEEE Trans. Commun., 2008, to appear.

[18] R. Chemaly, K. Letaief, and D. Zeghlache, "Adaptive resource allocation for multiuser MIMO/OFDM networks based on partial channel state information," in Proc. IEEE Global Telecomm. Conf., vol. 6, St. Louis, MO, Nov. 2005, pp. 1662-1672.

[19] P. Svedman, S. K. Wilson, L. J. Cimini, and B. Ottersten, "Opportunistic beamforming and scheduling for OFDMA systems," IEEE Trans. Commun., vol. 55, no. 5, pp. 941-952, May 2007.

[20] J. Oh and J. M. Cioffi, "Sub-band rate and power control for wireless OFDM systems," in Proc. IEEE Semi-Annual Veh. Technol. Conf., vol. 3 , Los Angeles, CA, Sep. 2004, pp. 2011-2014. 